\title{
CHARACTERIZATION OF GENERIC PROJECTIVE SPACE BUNDLES AND ALGEBRAICITY OF FOLIATIONS
}

\author{
CAROLINA ARAUJO AND STÉPHANE DRUEL
}

\begin{abstract}
In this paper we consider various notions of positivity for distributions on complex projective manifolds. We start by analyzing distributions having big slope with respect to curve classes, obtaining characterizations of generic projective space bundles in terms of movable curve classes. We then apply this result to investigate algebraicity of leaves of foliations, providing a lower bound for the algebraic rank of a foliation in terms of invariants measuring positivity. We classify foliations attaining this bound, and describe those whose algebraic rank slightly exceeds this bound.
\end{abstract}

\section{CONTENTS}

1. Introduction

2. Foliations

2.1. Basic notions

2.2. Algebraically integrable foliations

2.3. Foliations defined by stability conditions

3. Characterization of generic projective space bundles

4. Bounding the algebraic rank

References

\section{INTRODUCTION}

The existence of sufficiently positive subsheaves of the tangent bundle of a complex projective manifold $X$ imposes strong restrictions on $X$. In particular, several special varieties can be characterized by positivity properties of their tangent bundle. Early results in this direction include Kobayashi and Ochiai's characterizations of projective spaces and hyperquadrics KO73 and Mori's characterization of projective spaces [Mor79. There are many ways of measuring positivity of a torsion free sheaf. One way is to consider slopes with respect to movable curve classes $\alpha \in \operatorname{Mov}(X)$. We refer to Section 2.3 for the notion of slope and its properties. When $\alpha$ is an ample class, we have the following characterization of projective spaces due to Höring.

Theorem 1.1. Let $X$ be an $n$-dimensional complex normal projective variety, $\mathscr{L}$ an ample line bundle on $X$, and $\mathscr{F} \subseteq T_{X}$ a distribution. Set $\alpha:=\left[\mathscr{L}^{n-1}\right] \in \operatorname{Mov}(X)$. If $\mu_{\alpha}\left(\mathscr{F} \otimes \mathscr{L}^{*}\right)>0$, then

- $(X, \mathscr{L}) \cong\left(\mathbb{P}^{n}, \mathscr{O}_{\mathbb{P}^{n}}(1)\right)($ Hör14 , Theorem 1.1]), and

- $\mathscr{F}=T_{\mathbb{P}^{n}}($ ADK08, Theorem 1.3]).

Projective space bundles provide counter-examples to the statement of Theorem 1.1 if we replace the ample class $\alpha=\left[\mathscr{L}^{n-1}\right]$ with more general movable curve classes. More precisely, let $Y$ be a complex projective manifold, and $\mathscr{E}$ an ample vector bundle of rank $r+1 \geqslant 2$ on $Y$. Consider the projectivization $X=\mathbb{P}_{Y}(\mathscr{E})$, with tautological line bundle $\mathscr{O}_{X}(1)$ and natural morphism $\pi: X \rightarrow Y$. Let $\alpha \in \operatorname{Mov}(X)$ be the class of a line contained in a fiber of $\pi$. Then $\mu_{\alpha}\left(T_{X / Y} \otimes \mathscr{O}_{X}(-1)\right)=\frac{1}{r}>0$. So there is an open neighborhood $U \subset \operatorname{Mov}(X)$ of $\alpha$ such that $\mu_{\beta}\left(T_{X / Y} \otimes \mathscr{O}_{X}(-1)\right)>0$ for every $\beta \in U$. The following characterization of generic $\mathbb{P}^{r}$-bundles shows that these are all the new examples that arise when the ample class $\alpha=\left[\mathscr{L}^{n-1}\right]$ is replaced with an arbitrary movable class $\alpha \in \operatorname{Mov}(X)$ in Theorem 1.1. By a generic $\mathbb{P}^{r}$-bundle we mean an almost proper dominant map $X \rightarrow Y$ to a normal projective variety $Y$ with general fiber isomorphic to $\mathbb{P}^{r}$.

2010 Mathematics Subject Classification. 14M22, 14J40, 37F75. 
Theorem 1.2. Let $X$ be a normal $\mathbb{Q}$-factorial complex projective variety, and $\mathscr{L}$ an ample line bundle on $X$. If $\mu_{\alpha}^{\max }\left(T_{X} \otimes \mathscr{L}^{*}\right)>0$ for some movable curve class $\alpha \in \operatorname{Mov}(X)$, then $X$ is a generic $\mathbb{P}^{r}$-bundle for some positive integer $r$.

Theorem 1.2 follows from the more refined statement in Theorem 3.1 .

Corollary 1.3. Let $X$ be a normal $\mathbb{Q}$-factorial complex projective variety, and suppose that $X$ is not a generic $\mathbb{P}^{r}$-bundle for any positive integer $r$. Let $\mathscr{L}$ be an ample line bundle on $X$. Then, for any positive integer $m$ and any torsion-free quotient $\left(\Omega_{X}^{1} \otimes \mathscr{L}\right)^{\otimes m} \rightarrow \mathscr{Q}$ of positive rank, $\operatorname{det}(\mathscr{Q})$ is pseudo-effective.

Next we apply these results to investigate algebraicity of leaves of holomorphic foliations on complex projective manifolds.

A central problem in the theory of holomorphic foliations is to find conditions that guarantee the existence of algebraic leaves. Algebraic leaves of holomorphic foliations correspond to algebraic solutions of complex differential equations. It has been noted that positivity of foliations on complex projective varieties tend to improve algebraicity of leaves ([CLN96], Bos01, BM16, CCP15b, [AD13, LPT13, AD14, [AD16], AD17, CP15a, Dru17b, and [Dru17a]). In order to measure algebraicity of leaves, we introduce the algebraic rank. The algebraic rank $r^{a}(\mathscr{F})$ of a holomorphic foliation $\mathscr{F}$ on a complex algebraic variety $X$ is the maximum dimension of an algebraic subvariety through a general point of $X$ that is tangent to $\mathscr{F}$. These maximal algebraic subvarieties tangent to $\mathscr{F}$ are the leaves of a subfoliation $\mathscr{F}^{a} \subseteq \mathscr{F}$, the algebraic part of $\mathscr{F}$ (see Definition 2.4).

In a series of papers, we have addressed Fano foliations (AD13, AD14, AD16] and AD17]). These are holomorphic foliations $\mathscr{F}$ on complex projective varieties with ample anti-canonical class $-K_{\mathscr{F}}$. For a Fano foliation $\mathscr{F}$ on a complex projective manifold $X$, a rough measure of positivity is the index $\iota(\mathscr{F})$, which is the largest integer dividing $-K_{\mathscr{F}}$ in $\operatorname{Pic}(X)$. Our works on Fano foliations with high index indicated that the larger is the index, the larger is the algebraic rank of the Fano foliation. Now we investigate this relation between positivity and algebraicity of leaves for a wider class of foliations than Fano foliations.

We first introduce a new invariant measuring positivity for foliations with big anti-canonical class.

Definition 1.4. Let $X$ be a complex projective manifold, and $\mathscr{F}$ a holomorphic foliation on $X$ with big anti-canonical class $-K_{\mathscr{F}}$. The generalised index of $\mathscr{F}$ is defined as follows (see Lemma 4.1).

$\widehat{\iota}(\mathscr{F}):=\max \left\{t \in \mathbb{R} \mid-K_{\mathscr{F}} \equiv t A+E\right.$ where $A$ is an ample divisor and $E$ is a pseudo-effective $\mathbb{R}$-divisor $\}$.

We provide a lower bound for the algebraic rank in terms of the generalised index, and classify foliations attaining this bound. We refer to Definition 2.4 for the notions of pull-back and purely transcendental foliations.

Theorem 1.5. Let $X$ be an n-dimensional complex projective manifold, and $\mathscr{F} \subsetneq T_{X}$ a foliation on $X$ with big anti-canonical class. Then the algebraic rank and the generalised index of $\mathscr{F}$ satisfy

$$
r^{a}(\mathscr{F}) \geqslant \widehat{\iota}(\mathscr{F}) .
$$

Moreover, equality holds if and only if $X \cong \mathbb{P}^{n}$ and $\mathscr{F}$ is the linear pull-back of a purely transcendental foliation on $\mathbb{P}^{n-r^{a}(\mathscr{F})}$ with zero canonical class.

The following are immediate consequences of Theorem 1.5.

Corollary 1.6. Let $X$ be an n-dimensional complex projective manifold, and $\mathscr{F} \subsetneq T_{X}$ a Fano foliation of index $\iota(\mathscr{F})$ on $X$. Then the algebraic rank of $\mathscr{F}$ satisfies the following inequality.

$$
r^{a}(\mathscr{F}) \geqslant \iota(\mathscr{F}) .
$$

Moreover, equality holds if and only if $X \cong \mathbb{P}^{n}$ and $\mathscr{F}$ is the linear pull-back of a purely transcendental foliation on $\mathbb{P}^{n-r^{a}(\mathscr{F})}$ with zero canonical class.

Corollary 1.7. Let $X$ be an $n$-dimensional complex projective manifold, $L$ an ample divisor on $X$, and $\mathscr{F} \subsetneq T_{X}$ a foliation of rank $r$ on $X$. Suppose that $-K_{\mathscr{F}}-r L$ is pseudo-effective. Then $\left(X, \mathscr{O}_{X}(L)\right) \cong$ $\left(\mathbb{P}^{n}, \mathscr{O}_{\mathbb{P}^{n}}(1)\right)$, and $\mathscr{F}$ is induced by a linear projection of $\mathbb{P}^{n}$.

Finally, we address foliations $\mathscr{F}$ whose algebraic rank and generalised index satisfy $r^{a}(\mathscr{F}) \leqslant \widehat{\iota}(\mathscr{F})+1$. 
Theorem 1.8. Let $X$ be a complex projective manifold, and $\mathscr{F} \subsetneq T_{X}$ a foliation on $X$ with big anti-canonical class. Suppose that the algebraic rank and the generalised index of $\mathscr{F}$ satisfy $r^{a}(\mathscr{F}) \leqslant \widehat{\imath}(\mathscr{F})+1$. Then the closure of a general leaf of $\mathscr{F}^{a}$ is rationally connected.

The following is an immediate consequence of Theorem 1.8

Corollary 1.9. Let $X$ be a complex projective manifold, and $\mathscr{F} \subsetneq T_{X}$ a Fano foliation of index $\iota(\mathscr{F})$ on $X$. Suppose that the algebraic rank of $\mathscr{F}$ satisfies $r^{a}(\mathscr{F})=\iota(\mathscr{F})+1$. Then the closure of a general leaf of $\mathscr{F}^{a}$ is rationally connected.

We remark that codimension 1 Fano foliations $\mathscr{F}$ of index $n-3$ on $n$-dimensional projective manifolds were classified in AD17. When $X \not \mathbb{P}^{n}$, then either $\mathscr{F}$ is algebraically integrable or $r^{a}(\mathscr{F})=\iota(\mathscr{F})+1$.

Notation and conventions. We always work over the field $\mathbb{C}$ of complex numbers. Varieties are always assumed to be irreducible. We denote by $X_{\mathrm{ns}}$ the nonsingular locus of a variety $X$. When $X$ is a normal variety, and $\mathscr{F}$ is a quasi-coherent sheaf of generic rank $r$ on $X$, we denote by $T_{X}$ the sheaf $\left(\Omega_{X}^{1}\right)^{*}$, and by $\operatorname{det}(\mathscr{F})$ the sheaf $\left(\wedge^{r} \mathscr{F}\right)^{* *}$. If $\mathscr{E}$ is a vector bundle on a variety $X$, we denote by $\mathbb{P}_{X}(\mathscr{E})$ the Grothendieck projectivization $\operatorname{Proj}_{X}\left(\mathrm{~S}^{\bullet} \mathscr{E}\right)$.

Acknowledgements. This project was supported by the Brazilian-French Network in Mathematics. Much of this work was developed during the authors' visits to IMPA and Institut Fourier. We would like to thank both institutions for their support and hospitality.

The authors would also like to thank the anonymous referees for their helpful and very detailed reports.

The first named author was partially supported by CNPq and Faperj Research Fellowships. The second named author was partially supported by the ALKAGE project (ERC grant $\mathrm{Nr} 670846,2015-2020$ ) and by the project Foliage of Agence Nationale de la Recherche (ANR-16-CE40-0008-01).

\section{Foliations}

2.1. Basic notions. Let $X$ be a normal variety.

Definition 2.1. A distribution on $X$ is a coherent saturated subsheaf $\mathscr{F} \subset T_{X}$. By saturated we mean that the quotient $T_{X} / \mathscr{F}$ is torsion-free.

The rank $r$ of $\mathscr{F}$ is the generic rank of $\mathscr{F}$. The codimension of $\mathscr{F}$ is defined as $q:=\operatorname{dim} X-r$.

The normal sheaf of $\mathscr{F}$ is the sheaf $\mathscr{N}_{\mathscr{F}}:=\left(T_{X} / \mathscr{F}\right)^{* *}$.

The canonical class $K_{\mathscr{F}}$ of $\mathscr{F}$ is any Weil divisor on $X$ such that $\mathscr{O}_{X}\left(-K_{\mathscr{F}}\right) \cong \operatorname{det}(\mathscr{F})$. We say that $\mathscr{F}$ is $\mathbb{Q}$-Gorenstein if $K_{\mathscr{F}}$ is a $\mathbb{Q}$-Cartier divisor.

Definition 2.2. A foliation on $X$ is a distribution $\mathscr{F} \subset T_{X}$ that is closed under the Lie bracket.

Let $X^{\circ} \subset X_{\mathrm{ns}}$ be the maximal open subset such that $\mathscr{F}_{\mid X_{\mathrm{ns}}}$ is a subbundle of $T_{X_{\mathrm{ns}}}$. By Frobenius' Theorem, through any point of $X^{\circ}$ there is a maximal connected and immersed holomorphic submanifold $L \subset X^{\circ}$ such that $T_{L}=\mathscr{F} \mid L$. We say that such $L$ is a leaf of $\mathscr{F}$. A leaf is called algebraic if it is open in its Zariski closure.

2.3. To a codimension $q$ distribution $\mathscr{F}$ on a normal variety $X$, one naturally associates a unique (up to scaling) twisted $q$-form $\omega_{\mathscr{F}} \in H^{0}\left(X, \Omega_{X}^{q} \otimes \operatorname{det}\left(\mathscr{N}_{\mathscr{F}}\right)\right)$. This form does not vanish in codimension 1 , and completely determines the distribution $\mathscr{F}$. (See for instance [AD16, Paragraph 3.5] for details.)

Definition 2.4 (The algebraic and transcendental parts of a foliation [AD14, Definition 2]). Let $\mathscr{F}$ be a foliation of rank $r$ on a normal variety $X$. There exist a normal variety $Y$, unique up to birational equivalence, a dominant rational map with connected fibers $\varphi: X \rightarrow Y$, and a foliation $\mathscr{G}$ on $Y$ such that the following conditions hold (see [LPT11, Section 2.3]).

(1) $\mathscr{G}$ is purely transcendental, i.e., there is no positive-dimensional algebraic subvariety through a general point of $Y$ that is tangent to $\mathscr{G}$.

(2) $\mathscr{F}$ is the pull-back of $\mathscr{G}$ via $\varphi$. This means the following. Let $X^{\circ} \subset X$ and $Y^{\circ} \subset Y$ be smooth open subsets such that $\varphi$ restricts to a smooth morphism $\varphi^{\circ}: X^{\circ} \rightarrow Y^{\circ}$. Then $\mathscr{F}_{\mid X^{\circ}}=\left(d \varphi^{\circ}\right)^{-1}\left(\mathscr{G}_{\mid Y^{\circ}}\right)$. In this case we write $\mathscr{F}=\varphi^{-1} \mathscr{G}$.

The foliation $\mathscr{F}^{a}$ on $X$ induced by $\varphi$ is called the algebraic part of $\mathscr{F}$, and its rank is the algebraic rank of $\mathscr{F}$, which we denote by $r^{a}$. When $r^{a}=r$, we say that $\mathscr{F}$ is algebraically integrable. The foliation $\mathscr{G} \subset T_{Y}$ is called the transcendental part of $\mathscr{F}$. 
Next we relate the canonical class of a foliation with those of its algebraic and transcendental parts. For that we introduce some notation.

Definition 2.5. Let $\pi: X \rightarrow Y$ be a dominant morphism of normal varieties.

Let $D$ be a Weil $\mathbb{Q}$-divisor on $Y$. If $\pi: X \rightarrow Y$ is equidimensional, we define the pull-back $\pi^{*} D$ of $D$ to be the unique $\mathbb{Q}$-divisor on $X$ whose restriction to $\pi^{-1}\left(Y_{\mathrm{ns}}\right)$ is $\left(\pi_{\mid \pi^{-1}\left(Y_{\mathrm{ns}}\right)}\right)^{*} D_{\mid \pi^{-1}\left(Y_{\mathrm{ns}}\right)}$. This agrees with the usual pull-back if $D$ is $\mathbb{Q}$-Cartier.

We define the ramification divisor $R(\pi)$ of $\pi$ as follows. Let $Y^{\circ} \subset Y$ be a dense open subset such that $\operatorname{codim}\left(Y \backslash Y^{\circ}\right) \geqslant 2$ and $\pi$ restricts to an equidimensional morphism $\pi^{\circ}: X^{\circ}=\pi^{-1}\left(Y^{\circ}\right) \rightarrow Y^{\circ}$. Set

$$
R\left(\pi^{\circ}\right)=\sum_{D^{\circ}}\left(\left(\pi^{\circ}\right)^{*} D^{\circ}-\left(\left(\pi^{\circ}\right)^{*} D^{\circ}\right)_{\text {red }}\right),
$$

where $D^{\circ}$ runs through all prime divisors on $Y^{\circ}$. Then $R(\pi)$ is the Zariski closure of $R\left(\pi^{\circ}\right)$ in $X$.

Assume that either $K_{Y}$ is $\mathbb{Q}$-Cartier, or that $\pi$ is equidimensional. We define the relative canonical divisor of $X$ over $Y$ as $K_{X / Y}:=K_{X}-\pi^{*} K_{Y}$.

2.6 (The canonical class of a pull-back foliation). Let $\pi: X \rightarrow Y$ be a dominant morphism with connected fibers between normal varieties, $\mathscr{G}$ a foliation on $Y$, and $\mathscr{F}=\pi^{-1} \mathscr{G} \subset T_{X}$ its pull-back via $\pi$, as in Definition 2.4 (2). Assume that $\pi$ is equidimensional. Let $\left\{B_{i}\right\}_{i \in I}$ be the (possibly empty) set of prime divisors on $Y$ contained in the set of critical values of $\pi$ and invariant by $\mathscr{G}$. A straightforward computation shows that

$$
K_{\mathscr{F}}=\pi^{*} K_{\mathscr{G}}+K_{X / Y}-\sum_{i \in I}\left(\pi^{*} B_{i}-\left(\pi^{*} B_{i}\right)_{\mathrm{red}}\right) .
$$

In particular, if $\mathscr{F}$ is induced by $\pi$, then (2.1) reads

$$
K_{\mathscr{F}}=K_{X / Y}-R(\pi) .
$$

Remark 2.7. Let $\varphi: X \rightarrow Y$ be a dominant rational map with connected fibers between normal varieties, and $\mathscr{F}$ a foliation on $X$. Suppose that the general fiber of $\varphi$ is tangent to $\mathscr{F}$. This means that, for a general point $x$ on a general fiber $F$ of $\varphi$, the linear subspace $\mathscr{F}_{x} \subset T_{x} X$ determined by the inclusion $\mathscr{F} \subset T_{X}$ contains $T_{x} F$. Then, by [AD13, Lemma 6.7], there is a foliation $\mathscr{G}$ on $Y$ such that $\mathscr{F}=\varphi^{-1} \mathscr{G}$. We remark that this is not true in general if $\mathscr{F}$ is just a distribution.

2.8 (Restricting foliations to subvarieties). Let $X$ be a smooth variety, and $\mathscr{F}$ a codimension $q$ foliation on $X$. Let $Z$ be a smooth subvariety with $\operatorname{dim} Z \geqslant q$. Suppose that $Z$ is generically transverse to $\mathscr{F}$. This means that the associated twisted $q$-form $\omega_{\mathscr{F}} \in H^{0}\left(X, \Omega_{X}^{q} \otimes \operatorname{det}\left(\mathscr{N}_{\mathscr{F}}\right)\right)$ restricts to a nonzero twisted $q$-form on $Z$, and so $\mathscr{F}$ induces a foliation $\mathscr{F}_{Z}$ of codimension $q$ on $Z$. Then there is an effective divisor $B$ on $Z$ such that $\mathscr{F}_{Z}$ corresponds to a twisted $q$-form in $H^{0}\left(Z, \Omega_{Z}^{q} \otimes \operatorname{det}\left(\mathscr{N}_{\mathscr{F}}\right)_{\mid Z}(-B)\right)$ non vanishing in codimension 1. A straightforward computation shows that

$$
K_{\mathscr{F}_{Z}}+K_{X \mid Z}=K_{\mathscr{F} \mid Z}+K_{Z}-B .
$$

When $Z$ is a general hyperplane section, we show that $B=0$.

Lemma 2.9. Let $X \subset \mathbb{P}^{N}$ be a smooth projective variety, and $\mathscr{F}$ a foliation of codimension $q \leqslant \operatorname{dim} X-2$ on $X$. Let $H \subset \mathbb{P}^{N}$ be a general hyperplane. Then $\mathscr{F}$ induces a foliation $\mathscr{F}_{X \cap H}$ of codimension $q$ on $X \cap H$ with

$$
K_{\mathscr{F}_{X \cap H}}=\left(K_{\mathscr{F}}+H\right)_{\mid X \cap H} \cdot
$$

Proof. Let $H \subset \mathbb{P}^{N}$ be a general hyperplane, and set $Z:=X \cap H$. Notice that $Z$ is smooth and that $Z$ is generically transverse to $\mathscr{F}$. Let $B$ be the effective divisor on $Z$ introduced in 2.8, If $B_{1}$ is a prime divisor on $Z$, then $B_{1} \subset \operatorname{Supp}(B)$ if and only if $\mathscr{F}$ is tangent to $H$ at a general point of $B_{1}$.

Let $X^{\circ} \subset X$ be the open subset where $\mathscr{F}$ is a subbundle of $T_{X}$, and consider the incidence subset

$$
I^{\circ}=\left\{(x, H) \in X^{\circ} \times\left(\mathbb{P}^{N}\right)^{*} \mid x \in H \text { and } \mathscr{F}_{x} \subset T_{x} H\right\} .
$$

An easy dimension count gives that $\operatorname{dim} I^{\circ}=\operatorname{dim} X+N-(\operatorname{dim} X-q)-1 \leqslant \operatorname{dim} X+N-3$. It follows that if $H$ is general, then $B=0$. 
2.2. Algebraically integrable foliations. Throughout this subsection, we let $X$ be a normal projective variety, and $\mathscr{F}$ a $\mathbb{Q}$-Gorenstein algebraically integrable foliation on $X$.

Definition 2.10 (AD14, Definition 3.11]). Let $i: F \rightarrow X$ be the normalization of the closure of a general leaf of $\mathscr{F}$. There is a canonically defined effective $\mathbb{Q}$-divisor $\Delta_{F}$ on $F$ such that $K_{F}+\Delta_{F} \sim_{\mathbb{Q}} i^{*} K_{\mathscr{F}}$. The pair $\left(F, \Delta_{F}\right)$ is called a general log leaf of $\mathscr{F}$.

In the setup of Definition 2.10, we often write $L_{\mid F}$ for the pull-back $i^{*} L$ of a Cartier divisor $L$ on $X$.

2.11 (The family of $\log$ leaves of $\mathscr{F}$ AD14, Lemma 3.9 and Remark 3.12]). There is a unique proper subvariety $Y^{\prime}$ of the Chow variety of $X$ whose general point parametrizes the closure of a general leaf of $\mathscr{F}$ (viewed as a reduced and irreducible cycle in $X$ ). Let $Y$ be the normalization of $Y^{\prime}$, and $Z \rightarrow Y^{\prime} \times X$ the normalization of the universal cycle, with induced morphisms:

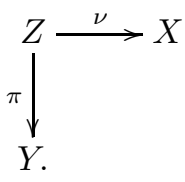

Then $\nu: Z \rightarrow X$ is birational and, for a general point $y \in Y, \nu\left(\pi^{-1}(y)\right) \subset X$ is the closure of a leaf of $\mathscr{F}$. We refer to the diagram (2.3) as the family of leaves of $\mathscr{F}$.

Let $\mathscr{F}_{Z}$ be the foliation on $Z$ induced by $\mathscr{F}\left(\right.$ or $\pi$ ). By (2.2),$K_{\mathscr{F}_{Z}}=K_{Z / Y}-R(\pi)$. Moreover, there is a canonically defined effective Weil $\mathbb{Q}$-divisor $\Delta$ on $Z$ such that

$$
K_{\mathscr{F}_{Z}}+\Delta=K_{Z / Y}-R(\pi)+\Delta \sim_{\mathbb{Q}} \nu^{*} K_{\mathscr{F}} .
$$

Note that $\Delta$ is $\nu$-exceptional since $\nu_{*} K_{\mathscr{F}_{Z}}=K_{\mathscr{F}}$.

Let $y \in Y$ be a general point, set $Z_{y}:=\pi^{-1}(y)$ and $\Delta_{y}:=\Delta_{\mid Z_{y}}$. Then $\left(Z_{y}, \Delta_{y}\right)$ coincides with the general $\log$ leaf $\left(F, \Delta_{F}\right)$ from Definition 2.10 .

We will need the following observation.

Lemma 2.12. Let $X$ be a smooth projective variety, and $\mathscr{F}$ an algebraically integrable foliation on $X$. In the setup of Paragraph 2.11 above, we have

$$
\operatorname{Supp}\left(\Delta_{y}\right)=\operatorname{Exc}(\nu) \cap Z_{y} .
$$

In particular, the singular locus of $Z_{y}$ is contained in $\operatorname{Supp}\left(\Delta_{y}\right)$.

Proof. Since $\Delta$ is $\nu$-exceptional and $\operatorname{Supp}\left(\Delta_{y}\right)=\operatorname{Supp}(\Delta) \cap Z_{y}$, we must have $\operatorname{Supp}\left(\Delta_{y}\right) \subset \operatorname{Exc}(\nu) \cap Z_{y}$.

To prove that $\operatorname{Exc}(\nu) \cap Z_{y} \subset \operatorname{Supp}\left(\Delta_{y}\right)$, we first reduce to the case when $\mathscr{F}$ has rank 1. Suppose that $\mathscr{F}$ has rank $r \geqslant 2$, and consider an embedding $X \subset \mathbb{P}^{N}$. Let $L \subset \mathbb{P}^{N}$ be a general linear subspace of codimension $r-1$. By Lemma 2.9, $\mathscr{F}$ induces a foliation by curves $\mathscr{F} X \cap L$ on $X \cap L$ with

$$
K_{\mathscr{F}_{X \cap L}}=\left(K_{\mathscr{F}}+(r-1) H\right)_{\mid X \cap L},
$$

where $H$ is a hyperplane in $\mathbb{P}^{N}$. Notice that the closure of a general leaf of $\mathscr{F}_{X \cap L}$ is $\nu\left(Z_{y}\right) \cap L$ for a general point $y \in Y$. Moreover, the general log leaf of $\mathscr{F}_{X \cap L}$ is $\left(Z_{y} \cap \nu^{-1}(L), \Delta_{y} \cap \nu^{-1}(L)\right)$. This follows from the definition of general $\log$ leaf, (2.5), and the usual adjunction formula. Let $E$ be an irreducible component of $\operatorname{Exc}(\nu)$ meeting $Z_{y}$. Since the restriction of $\nu$ to $E \cap Z_{y}$ is finite, we have $\operatorname{dim} \nu(E) \geqslant r-1$. In particular, $\nu(E) \cap L \neq \emptyset$. Moreover, $\operatorname{Exc}(\nu) \cap Z_{y} \subset \operatorname{Supp}\left(\Delta_{y}\right)$ if and only if $\operatorname{Exc}(\nu) \cap Z_{y} \cap \nu^{-1}(L) \subset \operatorname{Supp}\left(\Delta_{y}\right) \cap \nu^{-1}(L)$. Since $\operatorname{Exc}(\nu) \cap \nu^{-1}(L)=\operatorname{Exc}\left(\nu_{\mid \nu^{-1}(L)}\right)$, we may assume that $\mathscr{F}$ has rank 1 .

When $\mathscr{F}$ has rank 1, $\mathscr{F}$ is a line bundle on $X$ by [AD13, Remark 2.3] and [Har80, Proposition 1.9]. If $E$ is an irreducible component of $\operatorname{Exc}(\nu)$ that dominates $Y$, then $\nu(E)$ is contained in the singular locus of $\mathscr{F}$. Apply [Dru04, Lemme 1.2] (see also [AD13, Lemma 5.6]) to conclude that $E \cap Z_{y} \subset \operatorname{Supp}\left(\Delta_{y}\right)$, completing the proof of the lemma.

Corollary 2.13. Let $X$ be a smooth projective variety, and $\mathscr{F} \subsetneq T_{X}$ an algebraically integrable foliation on $X$, with general $\log$ leaf $\left(F, \Delta_{F}\right)$. Suppose that either $\rho(X)=1$, or $\mathscr{F}$ is a Fano foliation. Then $\Delta_{F} \neq 0$. 
Proof. Let the notation be as in Paragraph 2.11, and suppose that $\Delta_{F}=0$. It follows from Lemma 2.8 that no irreducible component of $\operatorname{Exc}(\nu)$ dominates $Y$. Hence, $\mathscr{F}$ is induced by a rational map $X \rightarrow Y$ that restricts to a smooth proper morphism on a dense open subset of $X$. This is impossible if $\rho(X)=1$. Moreover, $F$ is smooth, and in particular log canonical. On the other hand, an algebraically integrable Fano foliation whose general $\log$ leaf is log canonical has the special property that there is a common point in the closure of a general leaf (see [AD13, Proposition 5.3] and [AD16, Proposition 3.13]). We conclude that $\Delta_{F} \neq 0$.

We end this subsection with a consequence of [Hör12, Lemma 2.14] (see also CP15b, CP15a and Dru17b] for related results). Recall that a Weil $\mathbb{Q}$-divisor $D$ on a normal projective variety $X$ is said to be pseudo-effective if, for any ample divisor $L$ on $X$ and any rational number $\varepsilon>0$, there exists an effective Weil $\mathbb{Q}$-divisor $E$ such that $D+\varepsilon L \sim \mathbb{Q} E$.

Lemma 2.14. Let $X$ be a normal projective variety, $L$ an ample divisor on $X$, and $\mathscr{F}$ a $\mathbb{Q}$-Gorenstein algebraically integrable foliation on $X$. Let $F$ be the normalization of the closure of a general leaf of $\mathscr{F}$, and let $\nu_{F}: \widehat{F} \rightarrow F$ be a resolution of singularities. If $K_{\widehat{F}}+\nu_{F}^{*} L_{\mid F}$ is pseudo-effective, then so is $K_{\mathscr{F}}+L$.

Proof. Consider the family of leaves of $\mathscr{F}$ as in Paragraph 2.11

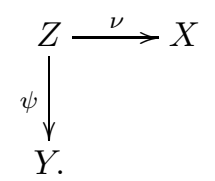

By Dru17b, Lemma 4.2], there exists a finite surjective morphism $\mu_{1}: Y_{1} \rightarrow Y$ with $Y_{1}$ normal and connected satisfying the following property. If $Z_{1}$ denotes the normalization of the product $Y_{1} \times_{Y} Z$, then the induced morphism $\psi_{1}: Z_{1} \rightarrow Y_{1}$ has reduced fibers over codimension one points in $Y_{1}$. Let $\nu_{1}: Z_{1} \rightarrow Z$ be the natural morphism. Let $\mu_{2}: Y_{2} \rightarrow Y_{1}$ be a resolution of singularities, and $Z_{2}$ a resolution of singularities of the product $Y_{2} \times_{Y_{1}} Z_{1}$, with natural morphism $\nu_{2}: Z_{2} \rightarrow Z_{1}$. We have a commutative diagram:



Let $F_{1} \cong F$ be a general fiber of $\psi_{1}$, and set $L_{1}:=\left(\nu \circ \nu_{1}\right)^{*} L$. Let $F_{2}$ be the fiber of $\psi_{2}: Z_{2} \rightarrow Y_{2}$ mapping to $F_{1}$, and set $L_{2}:=\nu_{2}^{*} L_{1}$.

By Paragraph 2.11, there is a canonically defined $\nu$-exceptional effective $\mathbb{Q}$-Weil divisor $\Delta$ on $Z$ such that

$$
K_{Z / Y}-R(\psi)+\Delta \sim_{\mathbb{Q}} \nu^{*} K_{\mathscr{F}},
$$

where $R(\psi)$ denotes the ramification divisor of $\psi$. Moreover, a straightforward computation shows that

$$
\nu_{1}^{*}\left(K_{Z / Y}-R(\psi)\right)=K_{Z_{1} / Y_{1}} .
$$

Suppose that $K_{\widehat{F}}+\nu_{F}^{*} L_{\mid F}$ is pseudo-effective. We may assume without loss of generality that the restriction of $\nu_{2}: Z_{2} \rightarrow Z_{1}$ to $F_{2}$ factors through $\widehat{F} \rightarrow F \cong F_{1}$. This implies that $K_{F_{2}}+L_{2 \mid F_{2}}$ is pseudo-effective as well. By [Hör12, Lemma 2.14], $K_{Z_{2} / Y_{2}}+L_{2}$ is also pseudo-effective. This implies that so is $\left(\nu \circ \nu_{1} \circ \nu_{2}\right)_{*}\left(K_{Z_{2} / Y_{2}}+L_{2}\right)$. Set $Z_{1}^{\circ}:=Z_{1} \backslash \nu_{2}\left(\operatorname{Exc}\left(\nu_{2}\right)\right)$ and $Y_{1}^{\circ}:=Y_{1} \backslash \mu_{2}\left(\operatorname{Exc}\left(\mu_{2}\right)\right)$. Since $\psi_{1}$ is equidimensional, we have $\psi_{1}\left(Z_{1}^{\circ}\right) \subset Y_{1}^{\circ}$.

Notice that codim $Y_{1} \backslash Y_{1}^{\circ} \geqslant 2$, codim $Z_{1} \backslash Z_{1}^{\circ} \geqslant 2$, and that $\nu_{2}$ (respectively, $\mu_{2}$ ) induces an isomorphism $\nu_{2}^{-1}\left(Z_{1} \backslash Z_{1}^{\circ}\right) \cong Z_{1} \backslash Z_{1}^{\circ}$ (respectively, $\left.\mu_{2}^{-1}\left(Y_{1} \backslash Y_{1}^{\circ}\right) \cong Y_{1} \backslash Y_{1}^{\circ}\right)$. It follows that $\left(\nu_{2}\right)_{*}\left(K_{Z_{2} / Y_{2}}+L_{2}\right)=K_{Z_{1} / Y_{1}}+L_{1}$. From (2.6) and (2.7), we conclude that

$$
\left(\nu \circ \nu_{1} \circ \nu_{2}\right)_{*}\left(K_{Z_{2} / Y_{2}}+L_{2}\right)=\operatorname{deg}\left(\nu_{1}\right)\left(K_{\mathscr{F}}+L\right)
$$

is pseudo-effective, completing the proof of the lemma. 
2.3. Foliations defined by stability conditions. Let $X$ be a normal projective variety, and $\mathscr{F} \subset T_{X}$ a foliation on $X$. Harder-Narasimhan filtrations of $\mathscr{F}$ allow one to construct subfoliations of $\mathscr{F}$ that inherit some of the positivity properties of $\mathscr{F}$ (see for instance AD13, Section 7]). However, the classical notion of slope-stability with respect to an ample line bundle is not flexible enough to be applied in many situations in birational geometry. The papers [CP11] and GKP16] extend a number of known results from the classical case to the setting where stability conditions are given by movable curve classes.

Definition 2.15. A curve class $\alpha \in \mathrm{N}_{1}(X)_{\mathbb{R}}$ is movable if $D \cdot \alpha \geqslant 0$ for all effective Cartier divisors $D$ on $X$. The set of movable classes is a closed convex cone $\operatorname{Mov}(X) \subset \mathrm{N}_{1}(X)_{\mathbb{R}}$, called the movable cone of $X$. If $X$ is smooth, then $\operatorname{Mov}(X)$ is the closure of the convex cone in $\mathrm{N}_{1}(X)_{\mathbb{R}}$ generated by classes of curves whose deformations cover a dense subset of $X$ by BDPP13.

2.16 (The Harder-Narasimhan filtration with respect to a movable curve class). Let $X$ be a normal, $\mathbb{Q}$ factorial, projective variety, $\alpha \in \mathrm{N}_{1}(X)_{\mathbb{R}}$ a movable curve class, and $\mathscr{F}$ a torsion-free sheaf of positive rank on $X$.

The slope of $\mathscr{F}$ with respect to $\alpha$ is the real number

$$
\mu_{\alpha}(\mathscr{F})=\frac{\operatorname{det}(\mathscr{F}) \cdot \alpha}{\operatorname{rank}(\mathscr{F})} .
$$

The sheaf $\mathscr{F}$ is $\alpha$-semistable if, for any subsheaf $\mathscr{E} \neq 0$ of $\mathscr{F}$, one has $\mu_{\alpha}(\mathscr{E}) \leqslant \mu_{\alpha}(\mathscr{F})$.

The maximal and minimal slopes of $\mathscr{F}$ with respect to $\alpha$ are defined by

$$
\begin{aligned}
& \mu_{\alpha}^{\max }(\mathscr{F}):=\sup \left\{\mu_{\alpha}(\mathscr{E}) \mid 0 \neq \mathscr{E} \subseteq \mathscr{F} \text { is a coherent subsheaf }\right\}, \\
& \mu_{\alpha}^{\min }(\mathscr{F}):=\inf \left\{\mu_{\alpha}(\mathcal{Q}) \mid \mathcal{Q} \neq 0 \text { is a torsion-free quotient of } \mathscr{F}\right\} .
\end{aligned}
$$

By [GKP16, Corollary 2.26], there exists a unique filtration of $\mathscr{F}$ by saturated subsheaves

$$
0=\mathscr{F}_{0} \subsetneq \mathscr{F}_{1} \subsetneq \cdots \subsetneq \mathscr{F}_{k}=\mathscr{F},
$$

with $\alpha$-semistable quotients $\mathcal{Q}_{i}=\mathscr{F}_{i} / \mathscr{F}_{i-1}$ such that $\mu_{\alpha}\left(\mathcal{Q}_{1}\right)>\mu_{\alpha}\left(\mathcal{Q}_{2}\right)>\cdots>\mu_{\alpha}\left(\mathcal{Q}_{k}\right)$. This filtration is called the Harder-Narasimhan filtration of $\mathscr{F}$.

Using the basic properties of slopes and the Harder-Narasimhan filtration, one can check that, for $1 \leqslant$ $i \leqslant k$,

$$
\mu_{\alpha}^{\min }\left(\mathscr{F}_{i}\right)=\mu_{\alpha}\left(\mathcal{Q}_{i}\right)=\mu_{\alpha}^{\max }\left(\mathscr{F} / \mathscr{F}_{i-1}\right)
$$

The sheaf $\mathscr{F}_{1}$ is called the maximal destabilizing subsheaf of $\mathscr{F}$.

Suppose that $\mu_{\alpha}^{\max }(\mathscr{F})>0$, and set $s:=\max \left\{1 \leqslant i \leqslant k \mid \mu_{\alpha}\left(\mathcal{Q}_{i}\right)>0\right\} \geqslant 1$. The positive part of $\mathscr{F}$ with respect to $\alpha$ is the sheaf $\mathscr{F}_{\alpha}^{+}:=\mathscr{F}_{s}$.

The following is a useful criterion for a subsheaf of a foliation to be a foliation. We include a proof for the reader's convenience.

Lemma 2.17 ([CP15a, Lemma 4.12]). Let $X$ be a normal, $\mathbb{Q}$-factorial, projective variety and $\mathscr{F} \subset T_{X}$ a foliation on $X$. Let $\mathscr{G} \subset \mathscr{F}$ be a saturated subsheaf and suppose that, for some movable curve class $\alpha \in \mathrm{N}_{1}(X)_{\mathbb{R}}$,

Then $\mathscr{G}$ is also a foliation on $X$.

$$
2 \mu_{\alpha}^{\min }(\mathscr{G})>\mu_{\alpha}^{\max }(\mathscr{F} / \mathscr{G}) .
$$

Proof. Note that $\mathscr{G}$ is saturated in $T_{X}$. Integrability of $\mathscr{G}$ is equivalent to the vanishing of the map $\wedge^{2} \mathscr{G} \rightarrow$ $\mathscr{F} / \mathscr{G}$ induced by the Lie bracket. This vanishing follows from the inequality

$$
\mu_{\alpha}^{\min }\left(\wedge^{2} \mathscr{G} / \text { Tors }\right)>\mu_{\alpha}^{\max }(\mathscr{F} / \mathscr{G}) \text {. }
$$

So it is enough to prove that

$$
\mu_{\alpha}^{\min }\left(\wedge^{2} \mathscr{G} / \text { Tors }\right) \geqslant 2 \mu_{\alpha}^{\min }(\mathscr{G}) .
$$

Observe $\left(\wedge^{2} \mathscr{G} / \text { Tors }\right)^{* *} \cong\left(\wedge^{2} \mathscr{G}\right)^{* *}$ is a direct summand of $(\mathscr{G} \otimes \mathscr{G})^{* *}$. Therefore, we have

$$
\begin{aligned}
\mu_{\alpha}^{\min }\left(\wedge^{2} \mathscr{G} / \text { Tors }\right) & =\mu_{\alpha}^{\min }\left(\left(\wedge^{2} \mathscr{G}\right)^{* *}\right) \\
& \geqslant \mu_{\alpha}^{\min }\left((\mathscr{G} \otimes \mathscr{G})^{* *}\right) \\
& =2 \mu_{\alpha}^{\min }(\mathscr{G})
\end{aligned}
$$

(by [GKP16, Theorem 4.2]), 
proving (2.9).

Corollary 2.18. In the setup of Paragraph 2.16, suppose that $\mathscr{F} \subset T_{X}$ is a foliation on $X$ with $\mu_{\alpha}^{\max }(\mathscr{F}) \geqslant 0$. Then $\mathscr{F}_{i} \subset T_{X}$ is also a foliation on $X$ whenever $\mu_{\alpha}\left(\mathcal{Q}_{i}\right) \geqslant 0$.

We end this subsection with a remarkable result of Campana and Păun concerning algebraic integrability of foliations.

Theorem 2.19. Let $X$ be a normal $\mathbb{Q}$-factorial projective variety, $\alpha \in \mathrm{N}_{1}(X)_{\mathbb{R}}$ a movable curve class, and $\mathscr{F} \subset T_{X}$ a foliation on $X$. Suppose that $\mu_{\alpha}^{\min }(\mathscr{F})>0$. Then $\mathscr{F}$ is algebraically integrable, and the closure of a general leaf is rationally connected.

In particular, if $\mathscr{F}$ is purely transcendental, then $K_{\mathscr{F}}$ is pseudo-effective.

Proof. Let $\nu: \widehat{X} \rightarrow X$ be a resolution of singularities. By GKP16, Proposition 2.7 and Remark 2.8], we have $\mu_{\alpha}^{\min }(\mathscr{F})=\mu_{\nu^{*} \alpha}^{\min }\left(\nu^{-1} \mathscr{F}\right)$, where $\nu^{*} \alpha \in \operatorname{Mov}(\widehat{X})$ is the numerical pull-back of $\alpha$. The claim now follows from [CP15a, Theorem 4.2] applied to $\nu^{-1} \mathscr{F}$.

The following is an immediate consequence of Corollary 2.18 and Theorem 2.19

Corollary 2.20. In the setup of Paragraph 2.16, suppose that $\mathscr{F} \subset T_{X}$ is a foliation on $X$ with $\mu_{\alpha}^{\max }(\mathscr{F})>0$. Then, for $1 \leqslant i \leqslant s, \mathscr{F}_{i} \subset T_{X}$ is an algebraically integrable foliation, and the closure of a general leaf is rationally connected.

\section{Characterization of Generic projective space Bundles}

Theorem 3.1. Let $X$ be a normal $\mathbb{Q}$-factorial projective variety, and $\mathscr{L}$ an ample line bundle on $X$. Suppose that $\mu_{\alpha}^{\max }\left(T_{X} \otimes \mathscr{L}^{*}\right)>0$ for some movable curve class $\alpha \in \operatorname{Mov}(X)$, and let $\mathscr{T}_{1}$ be the maximal destabilizing subsheaf of $T_{X}$ with respect to $\alpha$.

(1) Then $\mathscr{T}_{1}$ is induced by a generic $\mathbb{P}^{r_{1}}$-bundle structure $\pi_{1}: X \rightarrow Y_{1}$ on $X$, and $\mathscr{L}$ restricts to $\mathscr{O}_{\mathbb{P}_{1}}(1)$ on a general fiber of $\pi_{1}$. Moreover, $\mu_{\alpha}^{\max }\left(\left(T_{X} / \mathscr{T}_{1}\right) \otimes \mathscr{L}^{*}\right) \leqslant 0$.

(2) If $\mathscr{F}$ is a foliation on $X$ and $\mu_{\alpha}\left(\mathscr{F} \otimes \mathscr{L}^{*}\right)>0$, then $\mathscr{T}_{1} \subseteq \mathscr{F}$, and equality holds if and only if $\mathscr{F}$ is algebraically integrable.

Proof.

Step 1. Suppose first that $\mathscr{F}$ is an algebraically integrable foliation of $\operatorname{rank} r$, and $\mu_{\alpha}\left(\mathscr{F} \otimes \mathscr{L}^{*}\right)>0$ for some movable curve class $\alpha \in \operatorname{Mov}(X)$. We show that $\mathscr{F}$ is induced by a generic $\mathbb{P}^{r}$-bundle structure $\pi: X \rightarrow Y$ on $X$ and that $\mathscr{L}$ restricts to $\mathscr{O}_{\mathbb{P}^{r}}(1)$ on a general fiber of $\pi$.

Let $F$ be the normalization of the closure of a general leaf of $\mathscr{F}$, and let $\nu_{F}: \widehat{F} \rightarrow F$ be a resolution of singularities. Let $L$ be a divisor on $X$ such that $\mathscr{O}_{X}(L) \cong \mathscr{L}$. If $K_{\widehat{F}}+\operatorname{dim} F \cdot \nu_{F}^{*} L_{\mid F}$ is pseudo-effective, then so is $K_{\mathscr{F}}+\operatorname{dim} F \cdot L$ by Lemma 2.14 This is absurd since $-r \mu_{\alpha}\left(\mathscr{F} \otimes \mathscr{L}^{*}\right)=\left(K_{\mathscr{F}}+\operatorname{dim} F \cdot L\right) \cdot \alpha<0$ by assumption, proving that $K_{\widehat{F}}+\operatorname{dim} F \cdot \nu_{F}^{*} L_{\mid F}$ is not pseudo-effective. Apply [Hör14, Lemma 2.5] to conclude that $\left(F, \mathscr{L}_{\mid F}\right) \cong\left(\mathbb{P}^{r}, \mathscr{O}_{\mathbb{P} r}(1)\right)$. Consider the family of leaves of $\mathscr{F}$ as in Paragraph 2.11;

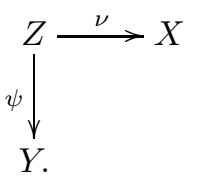

By [AD14, Proposition 4.10], we have $\left(Z, \nu^{*} \mathscr{L}\right) \cong\left(\mathbb{P}_{Y}(\mathscr{E}), \mathscr{O}_{\mathbb{P}_{Y}(\mathscr{E})}(1)\right)$ where $\mathscr{E}:=\psi_{*} \nu^{*} \mathscr{L}$.

Suppose that the exceptional set $\operatorname{Exc}(\nu)$ dominates $Y$ under $\psi$. Consider a resolution of singularities $\mu: \widehat{Y} \rightarrow Y$, and set $\widehat{Z}=\mathbb{P}_{\widehat{Y}}\left(\mu^{*} \mathscr{E}\right) \cong \widehat{Y} \times_{Y} Z$ with natural morphisms $\widehat{\psi}: \widehat{Z} \rightarrow \widehat{Y}$ and $\widehat{\nu}: \widehat{Z} \rightarrow X$. The exceptional set $\operatorname{Exc}(\widehat{\nu})$ also dominates $\widehat{Y}$ under $\widehat{\psi}$. Note that $\operatorname{Exc}(\widehat{\nu})$ has pure codimension one since $X$ is $\mathbb{Q}$ factorial. Let $\widehat{E}$ be an irreducible component of $\operatorname{Exc}(\widehat{\nu})$ dominating $\widehat{Y}$. Then $\mathscr{O}_{\widehat{Z}}(-\widehat{E}) \cong \mathscr{O}_{\mathbb{P}_{\widehat{Y}}\left(\mu^{*} \mathscr{E}\right)}(-k) \otimes \widehat{\psi}^{*} \mathscr{I}$ for some positive integer $k$, and some line bundle $\mathscr{I}$ on $\widehat{Y}$. In particular, $h^{0}\left(\widehat{Y}, \mathrm{~S}^{k} \mu^{*} \mathscr{E} \otimes \mathscr{I}^{*}\right) \geqslant 1$. Let $\widehat{\xi}$ be a divisor on $\widehat{Z}$ such that $\mathscr{O}_{\widehat{Z}}(\widehat{\xi}) \cong \mathscr{O}_{\mathbb{P}_{\widehat{Y}}\left(\mu^{*} \mathscr{E}\right)}(1)$. Notice that $\mathscr{O}_{\mathbb{P}_{\widehat{Y}}\left(\mu^{*} \mathscr{E}\right)}(1)$ is the pull-back of $\mathscr{O}_{\mathbb{P}_{Y}(\mathscr{E})}(1) \cong \nu^{*} \mathscr{L}$ under the natural morphism $\mathbb{P}_{\widehat{Y}}\left(\mu^{*} \mathscr{E}\right) \rightarrow \mathbb{P}_{Y}(\mathscr{E}) \cong Z$, and hence it is semi-ample. From Lemma 3.2 below, 
we conclude that $m_{0}\left(K_{\widehat{Z} / \widehat{Y}}+r \widehat{\xi}+s \widehat{E}\right)$ is effective for some positive integer $m_{0}$ and every sufficiently large integer $s$. This in turn implies that

$$
\widehat{\nu}_{*}\left(K_{\widehat{Z} / \widehat{Y}}+r \widehat{\xi}+s \widehat{E}\right)=K_{\mathscr{F}}+r L
$$

is pseudo-effective. Thus $\mu_{\alpha}\left(\mathscr{F} \otimes \mathscr{L}^{*}\right) \leqslant 0$, yielding a contradiction. This proves that the map $\pi: X \rightarrow Y$ induced by $\psi$ is almost proper, and so $\mathscr{F}$ is induced by a generic $\mathbb{P}^{r}$-bundle structure on $X$.

Step 2. We prove statement (1).

Let $0=\mathscr{T}_{0} \subsetneq \mathscr{T}_{1} \subsetneq \cdots \subsetneq \mathscr{T}_{k}=T_{X}$ be the Harder-Narasimhan filtration of $T_{X}$ with respect to $\alpha$, and set $\mathcal{Q}_{i}=\mathscr{T}_{i} / \mathscr{T}_{i-1}$. By Corollary $2.20, \mathscr{T}_{1}$ is an algebraically integrable foliation on $X$. Moreover, $\mu_{\alpha}\left(\mathscr{T}_{1} \otimes \mathscr{L}^{*}\right)>0$. From Step 1 applied to $\mathscr{T}_{1}$, we conclude that $\mathscr{T}_{1}$ is induced by a generic $\mathbb{P}^{r_{1}}$-bundle structure $\pi_{1}: X \rightarrow Y_{1}$ on $X$ and $\mathscr{L}$ restricts to $\mathscr{O}_{\mathbb{P}^{r_{1}}}(1)$ on a general fiber of $\pi_{1}$.

Suppose that $\mu_{\alpha}^{\max }\left(\left(T_{X} / \mathscr{T}_{1}\right) \otimes \mathscr{L}^{*}\right)=\mu_{\alpha}\left(\mathcal{Q}_{2} \otimes \mathscr{L}^{*}\right)>0$. By Corollary 2.20, $\mathscr{T}_{2}$ is an algebraically integrable foliation on $X$. By Step $1, \mathscr{T}_{2}$ is induced by a generic $\mathbb{P}^{r_{2}}$-bundle structure on $X$. This is impossible since $\mathbb{P}^{r_{2}}$ cannot admit a generic $\mathbb{P}^{r_{1}}$-bundle structure with $r_{1}<r_{2}$. Thus $\mu_{\alpha}\left(\mathscr{Q}_{2} \otimes \mathscr{L}^{*}\right) \leqslant 0$, proving (1).

Step 3. We prove statement (2).

Suppose that $\mathscr{F}$ is a foliation on $X$, and $\mu_{\alpha}\left(\mathscr{F} \otimes \mathscr{L}^{*}\right)>0$ for some movable curve class $\alpha \in \operatorname{Mov}(X)$. Let $\mathscr{F}_{1} \subset \mathscr{F}$ be the maximal destabilizing subsheaf of $\mathscr{F}$ with respect to $\alpha$. By Corollary 2.20, $\mathscr{F}_{1}$ is an algebraically integrable foliation on $X$. Moreover, we have $\mu_{\alpha}\left(\mathscr{F}_{1} \otimes \mathscr{L}^{*}\right)>0$. By Step $1, \mathscr{F}_{1}$ is induced by generic $\mathbb{P}^{s_{1}}$-bundle structure on $X$. Let $\mathscr{T}_{1} \subset T_{X}$ be as in Step 2. By GKP16, Corollary 2.17], $\mathscr{F}_{1} \subset \mathscr{T}_{1}$ since $\mu_{\alpha}^{\min }\left(\mathscr{F}_{1} \otimes \mathscr{L}^{*}\right)=\mu_{\alpha}\left(\mathscr{F}_{1} \otimes \mathscr{L}^{*}\right)>0$ and $\mu_{\alpha}^{\max }\left(\left(T_{X} / \mathscr{T}_{1}\right) \otimes \mathscr{L}^{*}\right) \leqslant 0$ by Step 2 . Hence, we must have $\mathscr{F}_{1}=\mathscr{T}_{1}$.

If $\mathscr{F}$ is algebraically integrable, then from Step 1 , we conclude that $\mathscr{F}_{1}=\mathscr{T}_{1}=\mathscr{F}$.

Recall that a vector bundle $\mathscr{E}$ on a normal projective variety $Y$ is said to be semi-ample if the line bundle $\mathscr{O}_{\mathbb{P}_{Y}(\mathscr{E})}(m)$ on $\mathbb{P}_{Y}(\mathscr{E})$ is generated by its global sections for some positive integer $m$.

Lemma 3.2. Let $Y$ be a normal projective variety, $\mathscr{M}$ a line bundle on $Y$, and $\mathscr{E}$ a semi-ample vector bundle on $Y$. Suppose that $h^{0}\left(Y, \mathrm{~S}^{k} \mathscr{E} \otimes \mathscr{M}\right) \geqslant 1$ for some integer $k \geqslant 1$. Then there exists an integer $m_{0} \geqslant 1$ such that $h^{0}\left(Y, \mathrm{~S}^{m_{0}(s k-1)} \mathscr{E} \otimes \operatorname{det}(\mathscr{E})^{\otimes m_{0}} \otimes \mathscr{M}^{\otimes m_{0} s}\right) \geqslant 1$ for every sufficiently large integer $s$.

Proof. Let $\mathscr{E}$ be a semi-ample vector bundle on $Y$. By [Fuj92, Corollary 1], the vector bundle $\mathscr{E}^{*} \otimes \operatorname{det}(\mathscr{E})$ is semi-ample. Let $m_{0}$ be a positive integer such that the line bundle $\mathscr{O}_{Z}\left(m_{0}\right)$ on $Z:=\mathbb{P}_{Y}\left(\mathscr{E}^{*} \otimes \operatorname{det}(\mathscr{E})\right)$ is generated by its global sections. Set $V:=H^{0}\left(Y, \mathrm{~S}^{m_{0}}\left(\mathscr{E}^{*} \otimes \operatorname{det}(\mathscr{E})\right)\right) \cong H^{0}\left(Z, \mathscr{O}_{Z}\left(m_{0}\right)\right)$, and consider the exact sequence

$$
0 \rightarrow \mathscr{K} \otimes \mathscr{O}_{Z}(m) \rightarrow V \otimes \mathscr{O}_{Z}(m) \rightarrow \mathscr{O}_{Z}\left(m_{0}+m\right) \rightarrow 0 .
$$

Pick a point $y$ on $Y$, and denote by $Z_{y}$ the fiber over $y$ of the natural morphism $Z \rightarrow Y$. Let $m_{1}$ be a positive integer such that $h^{1}\left(Z_{y}, \mathscr{K}_{\mid Z_{y}} \otimes \mathscr{O}_{Z_{y}}(m)\right)=0$ for $m \geqslant m_{1}$. Then the map

$$
V \otimes H^{0}\left(Z_{y}, \mathscr{O}_{Z_{y}}(m)\right) \rightarrow H^{0}\left(Z_{y}, \mathscr{O}_{Z_{y}}\left(m_{0}+m\right)\right)
$$

is surjective for every $m \geqslant m_{1}$, and thus the morphism

$$
V \otimes \mathrm{S}^{m}\left(\mathscr{E}^{*} \otimes \operatorname{det}(\mathscr{E})\right) \rightarrow \mathrm{S}^{m_{0}+m}\left(\mathscr{E}^{*} \otimes \operatorname{det}(\mathscr{E})\right)
$$

is generically surjective. This yields, for every $m \geqslant m_{1}$, an injective map of sheaves

$$
\mathrm{S}^{m_{0}+m}(\mathscr{E}) \hookrightarrow V^{*} \otimes \mathrm{S}^{m}(\mathscr{E}) \otimes \operatorname{det}(\mathscr{E})^{\otimes m_{0}} .
$$

Let $\mathscr{M}$ be a line bundle on $Y$, and $k$ a positive integer such that $h^{0}\left(Y, \mathrm{~S}^{k} \mathscr{E} \otimes \mathscr{M}\right) \geqslant 1$. Let $s$ be an integer such that $m:=m_{0}(s k-1) \geqslant m_{1}$. The $m_{0} s$-th power of a nonzero global section of $\mathrm{S}^{k} \mathscr{E} \otimes \mathscr{M}$ is a nonzero global section of $\mathrm{S}^{m_{0} s k} \mathscr{E} \otimes \mathscr{M}^{\otimes m_{0} s}$. Then 3.1 yields

$$
1 \leqslant h^{0}\left(Y, \mathrm{~S}^{m_{0} s k} \mathscr{E} \otimes \mathscr{M}^{\otimes m_{0} s}\right) \leqslant \operatorname{dim} V \cdot h^{0}\left(Y, \mathrm{~S}^{m_{0}(s k-1)} \mathscr{E} \otimes \operatorname{det}(\mathscr{E})^{\otimes m_{0}} \otimes \mathscr{M}^{\otimes m_{0} s}\right),
$$

completing the proof of the lemma. 
Proof of Corollary 1.3. Suppose that there is a positive integer $m$, an ample line bundle $\mathscr{L}$, a torsion-free quotient $\left(\Omega_{X}^{1} \otimes \mathscr{L}\right)^{\otimes m} \rightarrow \mathscr{Q}$ of positive rank, and a movable class $\alpha \in \operatorname{Mov}(X)$ such that $\mu_{\alpha}(\mathscr{Q})<0$. Then $\mu_{\alpha}^{\min }\left(\left(\Omega_{X}^{1} \otimes \mathscr{L}\right)^{\otimes m}\right)<0$. This implies that $\mu_{\alpha}^{\max }\left(\left(T_{X} \otimes \mathscr{L}^{*}\right)^{\otimes m}\right)>0$. Thus, by GKP16, Theorem 4.2] (see also [CP11, Proposition 6.1]), $\mu_{\alpha}^{\max }\left(T_{X} \otimes \mathscr{L}^{*}\right)>0$. Theorem 3.1 then implies that $X$ is a generic $\mathbb{P}^{r}$-bundle for some positive integer $r$.

\section{Bounding THE ALGEBRAIC RANK}

We start this section by justifying the definition of generalised index in Definition 1.4

Lemma 4.1. Let $X$ be a complex projective manifold, and $D$ a big divisor on $X$. Set

$$
\widehat{\iota}(D):=\sup \{t \in \mathbb{R} \mid D \equiv t A+E \text { where } A \text { is an ample divisor and } E \text { is a pseudo-effective } \mathbb{R} \text {-divisor }\} \text {. }
$$

Then there exists an ample divisor $A_{0}$ and a pseudo-effective $\mathbb{R}$-divisor $E_{0}$ on $X$ such that $D \equiv \widehat{\iota}(D) A_{0}+E_{0}$.

Proof. Set $t_{0}=\frac{\widehat{\imath}(D)}{2}<\infty$. Pick a real number $t \geqslant t_{0}$, an ample divisor $A_{t}$ and a pseudo-effective $\mathbb{R}$-divisor $E_{t}$ on $X$ such that $D \equiv t A_{t}+E_{t}$. Then $\frac{1}{t_{0}} D-A_{t}=\left(\frac{t}{t_{0}}-1\right) A_{t}+\frac{1}{t_{0}} E_{t}$ is pseudo-effective. In order to prove the lemma, it is enough to show that there are finitely many classes of integral effective divisors $B$ on $X$ such that $\frac{1}{t_{0}} D-B$ is pseudo-effective. Let $C_{1}, \ldots, C_{m}$ be movable curves on $X$ such that $\left[C_{1}\right], \ldots,\left[C_{m}\right]$ is a basis of $\mathrm{N}_{1}(X)_{\mathbb{R}}$. If $B$ is an effective divisor on $X$ such that $\frac{1}{t_{0}} D-B$ is pseudo-effective, then we have $0 \leqslant B \cdot C_{i} \leqslant \frac{1}{t_{0}} D \cdot C_{i}$. These inequalities define a compact set $\Delta \subset \mathrm{N}^{1}(X)_{\mathbb{R}}$. Since the set of classes of effective divisors is discrete in $\mathrm{N}^{1}(X)_{\mathbb{R}}$, the compact set $\Delta$ contains at most finitely many of these classes.

Next we show that the algebraic rank of a foliation is bounded from below by its generalised index, and classify foliations attaining this bound (Theorem 1.5). We fix the notation to be used in what follows.

Notation 4.2. Let $\mathscr{F} \subseteq T_{X}$ be a foliation with big anti-canonical class and generalised index $\widehat{\iota}$. Denote by $\mathscr{F}^{a}$ the algebraic part of $\mathscr{F}$, and by $r^{a}$ its algebraic rank. By Lemma 4.1 there is an ample divisor $L$ and a pseudo-effective $\mathbb{R}$-divisor $E$ such that $-K_{\mathscr{F}} \equiv \widehat{\iota} L+E$. Set $\mathscr{L}:=\mathscr{O}_{X}(L)$, and $\alpha_{0}=\left[\mathscr{L}^{n-1}\right] \in \operatorname{Mov}(X)$. For any movable curve class $\alpha \in \operatorname{Mov}(X)$, we denote by $\mathscr{F}_{\alpha}^{+}$the positive part of $\mathscr{F}$ with respect to $\alpha$, as defined in Paragraph 2.16, and by $r_{\alpha}=\operatorname{rank}\left(\mathscr{F}_{\alpha}^{+}\right)$its rank.

Proof of Theorem 1.5, We follow Notation 4.2, and assume that $\mathscr{F} \neq T_{X}$.

For any movable curve class $\alpha \in \operatorname{Mov}(X)$ such that $\mu_{\alpha}\left(\mathscr{F}_{\alpha}^{+} \otimes \mathscr{L}^{*}\right) \leqslant 0$, we have

$$
\begin{aligned}
\widehat{\iota} \mu_{\alpha}(\mathscr{L}) & \leqslant \operatorname{det}(\mathscr{F}) \cdot \alpha & \\
& \leqslant \operatorname{det}\left(\mathscr{F}_{\alpha}^{+}\right) \cdot \alpha & \left(\text { since } \mu_{\alpha}\left(\mathscr{F} / \mathscr{F}_{\alpha}^{+}\right) \leqslant 0\right) \\
& \leqslant r_{\alpha} \mu_{\alpha}(\mathscr{L}) & \left(\text { since } \mu_{\alpha}\left(\mathscr{F}_{\alpha}^{+} \otimes \mathscr{L}^{*}\right) \leqslant 0\right) \\
& \leqslant r^{a} \mu_{\alpha}(\mathscr{L}) & \left(\text { since } \mathscr{F}_{\alpha}^{+} \subset \mathscr{F}^{a} \text { by Theorem } 2.19\right) .
\end{aligned}
$$

By Theorem 1.1, we have $\mu_{\alpha_{0}}\left(\mathscr{F}_{\alpha_{0}}^{+} \otimes \mathscr{L}^{*}\right) \leqslant 0$ for the ample class $\alpha_{0}$, and (4.1) gives that $\widehat{\iota} \leqslant r^{a}$.

Suppose from now on that $\widehat{\iota}=r^{a}$. Then (4.1) shows that, for any movable curve class $\alpha \in \operatorname{Mov}(X) \operatorname{such}$ that $\mu_{\alpha}\left(\mathscr{F}_{\alpha}^{+} \otimes \mathscr{L}^{*}\right) \leqslant 0$ (in particular for $\alpha=\alpha_{0}$ ), we have $\mathscr{F}^{a}=\mathscr{F}_{\alpha}^{+}$, and $\mu_{\alpha}\left(\mathscr{F}^{a} \otimes \mathscr{L}^{*}\right)=0$. Moreover $E \cdot \alpha_{0}=0$, and thus $E=0$ and $-K_{\mathscr{F}} \equiv \widehat{\iota} L$.

Next we show that $\mu_{\alpha}\left(\mathscr{F}^{a} \otimes \mathscr{L}^{*}\right)=0$ for every $\alpha \in \operatorname{Mov}(X)$. Suppose that this is not the case. Since the ample class $\alpha_{0}$ lies in the interior of $\operatorname{Mov}(X)$ and $\mu_{\alpha_{0}}\left(\mathscr{F}^{a} \otimes \mathscr{L}^{*}\right)=0$, there must exist a class $\alpha \in \operatorname{Mov}(X)$ such that $\mu_{\alpha}\left(\mathscr{F}^{a} \otimes \mathscr{L}^{*}\right)>0$. By Theorem 3.1 there is a dense open subset $X^{\circ} \subset X$ and a $\mathbb{P}^{r^{a}}$-bundle structure $\pi^{\circ}: X^{\circ} \rightarrow Y^{\circ}$ such that $\mathscr{F}_{\mid X^{\circ}}^{a}=T_{X^{\circ} / Y^{\circ}}$, and $\mathscr{L}$ restricts to $\mathscr{O}_{\mathbb{P}^{r}}(1)$ on any fiber of $\pi^{\circ}$. By Remark 2.7, there is a foliation $\mathscr{G}$ on $Y^{\circ}$ such that $\mathscr{F} \mid X^{\circ}=\left(\pi^{\circ}\right)^{-1} \mathscr{G}$. By shrinking $Y^{\circ}$ if necessary, we may assume that $\mathscr{G}$ is locally free, and $\mathscr{F} / T_{X^{\circ} / Y^{\circ}} \cong \varphi^{*} \mathscr{G}$. Therefore, for a general fiber $F$ of $\pi^{\circ}$, we have

$$
\mathscr{O}_{\mathbb{P}^{r_{a}}}(\widehat{\iota}) \cong \operatorname{det}(\mathscr{F})_{\mid F} \cong \operatorname{det}\left(T_{X^{\circ} / Y^{\circ}}\right)_{\mid F} \cong \mathscr{O}_{\mathbb{P}^{r_{a}}}\left(r^{a}+1\right),
$$

contradicting our assumption that $\widehat{\iota}=r^{a}$. We conclude that $\mu_{\alpha}\left(\mathscr{F}^{a} \otimes \mathscr{L}^{*}\right)=0$ for every $\alpha \in \operatorname{Mov}(X)$.

This implies that $-K_{\mathscr{F} a} \equiv r^{a} L$, and hence $\mathscr{F}^{a}$ is an algebraically integrable Fano foliation. Let $\left(F, \Delta_{F}\right)$ be a general $\log$ leaf of $\mathscr{F}^{a}$, so that $-\left(K_{F}+\Delta_{F}\right) \equiv r^{a} L_{\mid F}$. By Corollary [2.13, $\Delta_{F} \neq 0$, and thus $K_{F}+$ $r^{a} L_{\mid F} \equiv-\Delta_{F}$ is not pseudo-effective. By [Hör14 Lemma 2.5], $\left(F, \mathscr{L}, \mathscr{O}_{F}\left(\Delta_{F}\right)\right) \cong\left(\mathbb{P}^{r^{a}}, \mathscr{O}_{\mathbb{P}^{a}}(1), \mathscr{O}_{\mathbb{P}^{r}}{ }^{a}(1)\right)$. In particular, $\left(F, \Delta_{F}\right)$ is $\log$ canonical. By [AD13, Proposition 5.3], there is a common point $x$ in the closure 
of a general leaf of $\mathscr{F}^{a}$. This implies that $X$ is covered by rational curves passing through $x$ having degree 1 with respect to $\mathscr{L}$. It follows that $X$ is a Fano manifold with Picard number $\rho(X)=1$, and hence $-K_{\mathscr{F} a} \equiv r^{a} L$ implies that $-K_{\mathscr{F} a} \sim r^{a} L$. By ADK08, Theorem 1.1], $X \cong \mathbb{P}^{n}$ and $\mathscr{F}^{a}$ is induced by a linear projection $\pi: \mathbb{P}^{n} \rightarrow \mathbb{P}^{n-r_{a}}$. By Remark 2.7 there is a foliation $\mathscr{G}$ on $\mathbb{P}^{n-r_{a}}$ such that $\mathscr{F}=\pi^{-1} \mathscr{G}$. By (2.1) and (2.2), outside the center of the linear projection $\pi: \mathbb{P}^{n} \rightarrow \mathbb{P}^{n-r_{a}}$, we have $\pi^{*} K_{\mathscr{G}} \sim K_{\mathscr{F}}-K_{\mathscr{F} a} \sim 0$, and thus $K_{\mathscr{G}} \sim 0$.

Finally, we describe foliations $\mathscr{F}$ whose algebraic rank slightly exceeds the generalised index, namely $\widehat{\iota}(\mathscr{F})<r^{a}(\mathscr{F}) \leqslant \widehat{\iota}(\mathscr{F})+1$. We start by addressing foliations $\mathscr{F}$ with $K_{\mathscr{F}} \equiv 0$ and algebraic rank $r^{a}=1$.

Lemma 4.3. Let $X$ be a complex projective manifold with Picard number $\rho(X)=1$, and let $\mathscr{F}$ be a foliation on $X$. Suppose that $K_{\mathscr{F}} \equiv 0$ and that $\mathscr{F}$ has algebraic rank 1 . Then the closure of a general leaf of the algebraic part of $\mathscr{F}$ is a rational curve.

Proof. There exist a normal projective variety $Y$, a dominant rational map $\pi: X \rightarrow Y$ of relative dimension 1 , and a purely transcendental foliation $\mathscr{G}$ on $Y$ such that $\mathscr{F}=\pi^{-1} \mathscr{G}$. Denote by $\mathscr{C}$ the foliation induced by $\pi$, i.e., the algebraic part of $\mathscr{F}$.

After replacing $Y$ with a birationally equivalent variety, we may assume that $Y$ is the family of leaves of $\mathscr{C}$. Let $X^{\circ} \subset X$ be an open subset with complement of codimension at least 2 such that $\pi$ restricts to an equidimensional morphism $\pi_{\mid X^{\circ}}: X^{\circ} \rightarrow Y$. By (2.1) and (2.2) applied to $\pi_{\mid X^{\circ}}$, there is an effective divisor $R$ on $X$ such that, on $X^{\circ}$, we have

$$
-K_{\mathscr{C}} \equiv \pi^{*} K_{\mathscr{G}}+R
$$

By Theorem 2.19, $K_{\mathscr{G}}$ is pseudo-effective, and hence so is $-K_{\mathscr{C}}$. If $K_{\mathscr{C}} \not \equiv 0$, then the lemma follows from Theorem 2.19] Suppose from now on that $K_{\mathscr{C}} \equiv 0$, and let $\left(F, \Delta_{F}\right)$ be a general $\log$ leaf of $\mathscr{C}$, so that $K_{F}+\Delta_{F} \equiv 0$. By Corollary 2.13, $\Delta_{F} \neq 0$. Thus $\operatorname{deg}\left(K_{F}\right)<0$, and hence $F \cong \mathbb{P}^{1}$.

Proof of Theorem 1.8. We follow Notation 4.2, and assume that $\widehat{\iota}<r^{a} \leqslant \widehat{\imath}+1$. We will show that the closure of a general leaf of $\mathscr{F}^{a}$ is rationally connected.

Step 1. Let $\alpha \in \operatorname{Mov}(X)$ be a movable curve class. If $\mu_{\alpha}\left(\mathscr{F}_{\alpha}^{+} \otimes \mathscr{L}^{*}\right)<0$, then (4.1) gives that $r^{a}-1 \leqslant \hat{\imath}<$ $r_{\alpha} \leqslant r^{a}$. Hence, $\mathscr{F}_{\alpha}^{+}=\mathscr{F}^{a}$. By Theorem 2.19, the closure of a general leaf of $\mathscr{F}^{a}$ is rationally connected. If $\mu_{\alpha}\left(\mathscr{F}_{\alpha}^{+} \otimes \mathscr{L}^{*}\right)=0$, then (4.1) gives that $r^{a}-1 \leqslant \widehat{\imath} \leqslant r_{\alpha} \leqslant r^{a}$, and hence $\mathscr{F}_{\alpha}^{+}$has codimension at most 1 in $\mathscr{F}^{a}$. If $\mathscr{F}_{\alpha}^{+}=\mathscr{F}^{a}$, then, as before, the closure of a general leaf of $\mathscr{F}^{a}$ is rationally connected. Suppose that $\mathscr{F}_{\alpha}^{+}$has codimension 1 in $\mathscr{F}^{a}$. Then $\widehat{\iota}=r^{a}-1$. Let $\beta \in \operatorname{Mov}(X)$ be another movable curve class. If $\mathscr{F}_{\beta}^{+} \not \subset \mathscr{F}_{\alpha}^{+}$, then $\mathscr{F}_{\alpha}^{+}+\mathscr{F}_{\beta}^{+}=\mathscr{F}^{a}$ and Theorem 2.19 applied to both $\alpha$ and $\beta$ gives that the closure of a general leaf of $\mathscr{F}^{a}$ is rationally connected.

Therefore, from now on we may assume the following.

Additional assumption: For any $\alpha \in \operatorname{Mov}(X), \mu_{\alpha}\left(\mathscr{F}_{\alpha}^{+} \otimes \mathscr{L}^{*}\right) \geqslant 0$. If $\alpha \in \operatorname{Mov}(X)$ is such that $\mu_{\alpha}\left(\mathscr{F}_{\alpha}^{+} \otimes\right.$ $\left.\mathscr{L}^{*}\right)=0$, then $\mathscr{F}_{\alpha}^{+}$has codimension 1 in $\mathscr{F}^{a}$, and $\mathscr{F}_{\beta}^{+} \subset \mathscr{F}_{\alpha}^{+}$for any $\beta \in \operatorname{Mov}(X)$.

By Theorem 1.1, we have $\mu_{\alpha_{0}}\left(\mathscr{F}_{\alpha_{0}}^{+} \otimes \mathscr{L}^{*}\right) \leqslant 0$ for the ample class $\alpha_{0}$. The additional assumption and (4.1) for $\alpha=\alpha_{0}$ give that $r_{\alpha_{0}}=r^{a}-1=\widehat{\iota}, E=0$ and $-K_{\mathscr{F}} \equiv \widehat{\iota} L$.

Step 2. Suppose that $\mu_{\alpha}\left(\mathscr{F}_{\alpha}^{+} \otimes \mathscr{L}^{*}\right)=0$ for every $\alpha$ in a nonempty open subset $U$ of $\operatorname{Mov}(X)$. From the additional assumption, it follows that $\mathscr{F}_{\alpha}^{+}=\mathscr{F}_{\alpha_{0}}^{+}$and $\mu_{\alpha}\left(\mathscr{F}_{\alpha_{0}}^{+} \otimes \mathscr{L}^{*}\right)=0$ for every $\alpha \in U$. Thus $-K_{\mathscr{F}_{\alpha_{0}}} \equiv r_{\alpha_{0}} L$. By Theorem 1.5, $X \cong \mathbb{P}^{n}$, and $\mathscr{F}_{\alpha_{0}}^{+}$is induced by a linear projection $\pi: \mathbb{P}^{n} \rightarrow \mathbb{P}^{n-r_{\alpha_{0}}}$. Since $X \cong \mathbb{P}^{n}$, we must have $-K_{\mathscr{F}} \sim \hat{\imath} L \sim-K_{\mathscr{F}_{\alpha_{0}}}$. By Remark 2.7 there is a foliation $\mathscr{G}$ on $\mathbb{P}^{n-r_{\alpha_{0}}}$ such

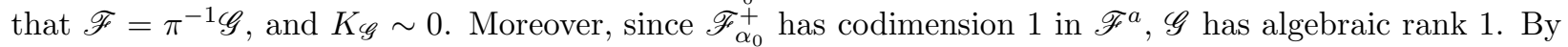
Lemma 4.3 the closure of a general leaf of the algebraic part of $\mathscr{G}$ is a rational curve, and thus the closure of a general leaf of $\mathscr{F}^{a}$ is rationally connected.

Step 3. By Step 2, we may assume that $\mu_{\alpha}\left(\mathscr{F}_{\alpha}^{+} \otimes \mathscr{L}^{*}\right)>0$ for some $\alpha \in \operatorname{Mov}(X)$. By Theorem 3.1, $\mathscr{F}_{\alpha}^{+}$is induced by a generic $\mathbb{P}^{r_{\alpha}}$-bundle structure $\pi_{\alpha}: X \rightarrow Y_{\alpha}$ on $X$, and $\mathscr{L}$ restricts to $\mathscr{O}_{\mathbb{P}^{r} \alpha}(1)$ on general fibers 
of $\pi_{\alpha}$. If necessary, we can replace $\alpha$ by the class of a line on a fiber of $\pi_{\alpha}$. As in (4.1), we have

$$
\begin{aligned}
\left(r^{a}-1\right)=\widehat{\iota} & =\operatorname{det}(\mathscr{F}) \cdot \alpha \\
& \leqslant \operatorname{det}\left(\mathscr{F}_{\alpha}^{+}\right) \cdot \alpha \\
& =\left(r_{\alpha}+1\right),
\end{aligned}
$$

and thus $r_{\alpha} \geqslant r^{a}-2$. Moreover, by Corollary 1.7, $-K_{\mathscr{F}_{\alpha}^{+}}-r_{\alpha} L$ is not pseudo-effective. Thus, there exists a nonempty open subset $V$ of $\operatorname{Mov}(X)$ such that, for every $\beta \in V, \mu_{\beta}\left(\mathscr{F}_{\alpha}^{+} \otimes \mathscr{L}^{*}\right)<0$. The additional assumption implies in particular that $\mathscr{F}_{\beta}^{+} \neq \mathscr{F}_{\alpha}^{+}$for every $\beta \in V$.

By Step 2, we may assume that $\mu_{\beta}\left(\mathscr{F}_{\beta}^{+} \otimes \mathscr{L}^{*}\right)>0$ for some $\beta \in V$. As before, $\mathscr{F}_{\beta}^{+}$is induced by a generic $\mathbb{P}^{r_{\beta}}$-bundle structure $\pi_{\beta}: X \rightarrow Y_{\beta}$ on $X, \mathscr{L}$ restricts to $\mathscr{O}_{\mathbb{P}^{r}}(1)$ on general fibers of $\pi_{\beta}$, and $r_{\beta} \geqslant r^{a}-2$. Notice that $\mathscr{F}_{\alpha}^{+} \cap \mathscr{F}_{\beta}^{+}=0$ and $\mathscr{F}_{\alpha}^{+}+\mathscr{F}_{\beta}^{+} \subset \mathscr{F}^{a}$. If $\mathscr{F}_{\alpha}^{+}+\mathscr{F}_{\beta}^{+}=\mathscr{F}^{a}$, then we conclude as before that the closure of a general leaf of $\mathscr{F}^{a}$ is rationally connected. So we may assume that $\mathscr{F}_{\alpha}^{+}+\mathscr{F}_{\beta}^{+} \subsetneq \mathscr{F}^{a}$. This can only happen if $\left(r_{\alpha}, r_{\beta}, r^{a}\right)=(1,1,3)$.

Let $H_{\alpha}$ and $H_{\beta}$ be the dominating unsplit families of rational curves on $X$ whose general members correspond to lines on fibers of $\pi_{\alpha}$ and $\pi_{\beta}$, respectively. Denote by $\pi^{\circ}: X^{\circ} \rightarrow T^{\circ}$ the $\left(H_{\alpha}, H_{\beta}\right)$-rationally connected quotient of $X$ (see for instance [AD13, 6.4] for this notion). By [ADK08, Lemma 2.2], we may assume that $\operatorname{codim} X \backslash X^{\circ} \geqslant 2, T^{\circ}$ is smooth, and $\pi^{\circ}$ has irreducible and reduced fibers. Applying Gro66, Corollaires 14.4.4 et 15.2.3], we see that $\pi^{\circ}$ is flat. This in turn implies that its fibers are Cohen-Macaulay since both $X$ and $T^{\circ}$ are smooth. By [AD13, Lemma 6.9], there is an inclusion $T_{X^{\circ} / T^{\circ}} \subset \mathscr{F}_{\mid X^{\circ}}^{a}$. If $T_{X^{\circ} / T^{\circ}}=\mathscr{F}_{\mid X^{\circ}}^{a}$, then the closure of a general leaf of $\mathscr{F}^{a}$ is rationally connected. So we may assume that $\operatorname{dim} T^{\circ}=\operatorname{dim} X^{\circ}-2$. By Remark 2.7, there is a foliation $\mathscr{G}^{\circ}$ on $T^{\circ}$ such that $\mathscr{F} \mid X^{\circ}=\pi^{\circ-1} \mathscr{G}^{\circ}$. By (2.1), we have

$$
-K_{X^{\circ} / T^{\circ}}=-K_{\mathscr{F} \mid X^{\circ}}+\pi^{\circ *} K_{\mathscr{G}} \circ .
$$

Since $\operatorname{codim} X \backslash X^{\circ} \geqslant 2$, a general complete intersection curve in $X$ is contained in $X \backslash X^{\circ}$. Let $C \rightarrow T^{\circ}$ be the normalization of a complete curve passing through a general point, denote by $X_{C}$ the fiber product $C \times_{T^{\circ}} X^{\circ}$, and by $\pi_{C}: X_{C} \rightarrow C$ the natural morphism. By [Gro65, Corollaire 5.12.4], $X_{C}$ satisfies Serre's condition $S_{2}$. On the other hand, $X_{C}$ is smooth in codimension 1 since the fibers of $\pi_{C}$ are reduced. It follows that $X_{C}$ is a normal variety by Serre's criterion for normality. We have

$$
-K_{X_{C} / C}=-K_{\mathscr{F} \mid X_{C}}+\pi_{C}{ }^{*} K_{\mathscr{G}} \circ \mid C .
$$

If $K_{\mathscr{G} \circ} \cdot C \geqslant 0$, then $-K_{X_{C} / C}$ is ample, contradicting [ADK08, Theorem 3.1]. Hence, $K_{\mathscr{G} \circ} \cdot C<0$, and [AD13, Proposition 7.5] implies that the closure of a general leaf of $\mathscr{F}$ is rationally connected.

Question 4.4. Is there a foliation $\mathscr{F}$ with $\widehat{\iota}(\mathscr{F}) \notin \mathbb{N}$ and $\widehat{\iota}(\mathscr{F})<r^{a}(\mathscr{F})<\widehat{\iota}(\mathscr{F})+1$ ? From the proof of Theorem 1.8, we see that in this case we must have $\mathscr{F}_{\alpha}^{+}=\mathscr{F}^{a}$ for every $\alpha \in \operatorname{Mov}(X)$ such that $\mu_{\alpha}\left(\mathscr{F}_{\alpha}^{+} \otimes\right.$ $\left.\mathscr{L}^{*}\right) \leqslant 0$.

\section{REFERENCES}

[AD13] Carolina Araujo and Stéphane Druel, On Fano foliations, Adv. Math. 238 (2013), 70-118.

[AD14] On codimension 1 del Pezzo foliations on varieties with mild singularities, Math. Ann. 360 (2014), no. 3-4, 769-798.

[AD16] , On Fano foliations 2., Foliation theory in algebraic geometry. Proceedings of the conference, New York, NY, USA, September 3-7, 2013, Cham: Springer, 2016, pp. 1-20.

[AD17] _ Codimension 1 Mukai foliations on complex projective manifolds, J. Reine Angew. Math. 727 (2017), 191-246.

[ADK08] Carolina Araujo, Stéphane Druel, and Sándor J. Kovács, Cohomological characterizations of projective spaces and hyperquadrics, Invent. Math. 174 (2008), no. 2, 233-253.

[BDPP13] Sébastien Boucksom, Jean-Pierre Demailly, Mihai Păun, and Thomas Peternell, The pseudo-effective cone of a compact Kähler manifold and varieties of negative Kodaira dimension, J. Algebraic Geom. 22 (2013), no. 2, 201248.

[BM16] Fedor Bogomolov and Michael McQuillan, Rational curves on foliated varieties, Foliation theory in algebraic geometry, Simons Symp., Springer, Cham, 2016, pp. 21-51.

[Bos01] Jean-Benoît Bost, Algebraic leaves of algebraic foliations over number fields, Publ. Math. Inst. Hautes Études Sci. (2001), no. 93, 161-221.

[CLN96] D. Cerveau and A. Lins Neto, Irreducible components of the space of holomorphic foliations of degree two in $\mathbf{C P}(n)$, $n \geq 3$, Ann. of Math. (2) 143 (1996), no. 3, 577-612. 
[CP11] Frédéric Campana and Thomas Peternell, Geometric stability of the cotangent bundle and the universal cover of a projective manifold, Bull. Soc. Math. France 139 (2011), no. 1, 41-74, With an appendix by Matei Toma.

[CP15a] Frédéric Campana and Mihai Păun, Foliations with positive slopes and birational stability of orbifold cotangent bundles, Preprint arXiv:1508:0245v4, 2015.

[CP15b] Frédéric Campana and Mihai Păun, Orbifold generic semi-positivity: an application to families of canonically polarized manifolds, Ann. Inst. Fourier (Grenoble) 65 (2015), no. 2, 835-861.

[Dru04] Stéphane Druel, Caractérisation de l'espace projectif, Manuscripta Math. 115 (2004), no. 1, 19-30.

[Dru17a] _ A decomposition theorem for singular spaces with trivial canonical class of dimension at most five, Invent. Math., to appear. DOI : 10.1007/s00222-017-0748-y, 2017.

[Dru17b] Stéphane Druel, On foliations with nef anti-canonical bundle, Trans. Amer. Math. Soc. 369 (2017), no. 11, 77657787 .

[Fuj92] Tsuyoshi Fujiwara, Varieties of small Kodaira dimension whose cotangent bundles are semiample, Compositio Math. 84 (1992), no. 1, 43-52.

[GKP16] Daniel Greb, Stefan Kebekus, and Thomas Peternell, Movable curves and semistable sheaves, Internat. Math. Res. Notices 2016 (2016), no. 2, 536-570.

[Gro65] A. Grothendieck, Éléments de géométrie algébrique. IV. Étude locale des schémas et des morphismes de schémas. II, Inst. Hautes Études Sci. Publ. Math. (1965), no. 24, 231.

[Gro66] É Éléments de géométrie algébrique. IV. Étude locale des schémas et des morphismes de schémas. III, Inst. Hautes Études Sci. Publ. Math. (1966), no. 28, 255.

[Har80] Robin Hartshorne, Stable reflexive sheaves, Math. Ann. 254 (1980), no. 2, 121-176.

[Hör12] Andreas Höring, On a conjecture of Beltrametti and Sommese, J. Algebraic Geom. 21 (2012), no. 4, 721-751.

[Hör14] Twisted cotangent sheaves and a Kobayashi-Ochiai theorem for foliations, Ann. Inst. Fourier (Grenoble) 64 (2014), no. 6, 2465-2480.

[KO73] S. Kobayashi and T. Ochiai, Characterizations of complex projective spaces and hyperquadrics, J. Math. Kyoto Univ. 13 (1973), 31-47.

[LPT11] Frank Loray, Jorge Vitório Pereira, and Frédéric Touzet, Singular foliations with trivial canonical class, Preprint arXiv:1107.1538 5, 2011.

[LPT13] Frank Loray, Jorge Vitório Pereira, and Frédéric Touzet, Foliations with trivial canonical bundle on Fano 3-folds, Math. Nachr. 286 (2013), no. 8-9, 921-940.

[Mor79] S. Mori, Projective manifolds with ample tangent bundles, Ann. of Math. (2) 110 (1979), no. 3, 593-606.

Carolina Araujo: impa, Estrada Dona Castorina 110, Rio de Janeiro, 22460-320, Brazil

E-mail address: caraujo@impa.br

Stéphane Druel: Institut Fourier, UMR 5582 du CNRS, Université Grenoble Alpes, CS 40700, 38058 Grenoble CEDEX 9, France

E-mail address: stephane.druel@univ-grenoble-alpes.fr 